\title{
Candidate gene studies reveal that the WFS1 gene joins the expanding list of novel type 2 diabetes genes
}

\author{
J. Wasson • M. A. Permutt
}

Received: 18 December 2007 / Accepted: 19 December 2007 / Published online: 15 January 2008

(C) Springer-Verlag 2007

Keywords Gene polymorphisms · Genetic risk .

Type 2 diabetes

\author{
Abbreviations \\ ER endoplasmic reticulum \\ GWA genome-wide association \\ LD linkage disequilibrium \\ OR odds ratio \\ SNP single nucleotide polymorphism
}

\section{Wolfram syndrome}

Wolfram syndrome, originally described in 1938, is a rare, autosomal recessive disease that is characterised by young onset insulin-dependent diabetes, progressive sensorineural deafness, diabetes insipidus, autonomic nervous system dysfunction and, ultimately, brainstem atrophy and premature death [1]. The Wolfram gene (WFS1), which encodes wolframin, was mapped to chromosome $4 \mathrm{p}$ in families with multiple affected individuals [2], and cloned in 1998 [3]. Wolframin is a protein of 890 amino acids that is produced in a wide variety of tissues, most prominently in pancreatic beta cells and brain. Over 100 missense and non-sense mutations have been described patients. As these mutations

\footnotetext{
J. Wasson $\cdot$ M. A. Permutt $(\square)$

Division of Endocrinology, Metabolism, and Lipid Research, Department of Medicine, Washington University School of Medicine, 660 S. Euclid Ave., Campus Box 8127,

St Louis, MO 63110, USA

e-mail: apermutt@im.wustl.edu
}

are associated with a non-immune loss of beta cells and diabetes, the gene was subsequently evaluated in more common forms of diabetes.

\section{Do variants of WFS1 contribute to risk of type 2 diabetes?}

Variants of WFS1 have not convincingly been shown to contribute to the risk of type 1 diabetes [4]. The first study to address this question in type 2 diabetes sequenced DNA from 29 patients and uncovered 12 coding variants or single nucleotide polymorphisms (SNPs). These included five non-synonymous SNPs encoding amino acid changes [5]. The most abundant genetic variant alters the amino acid at position 611 from a histidine to an arginine (H611R). The arginine variant was present in $40 \%$ of diabetic cases and $45 \%$ of controls $(p<0.02)$, suggesting that the variant was protective from diabetes. There were no functional studies, and the sample size in this initial study was relatively small. These observations suggested that variation in WFS1 influences susceptibility to type 2 diabetes.

As beta cell dysfunction is a critical component of type 2 diabetes, a recent study genotyped 1,500 SNPs as markers for 84 beta cell candidate genes in a large number of UK Europid and Ashkenazi Jewish cases and controls [6]. Among all the genes examined, only SNPs in WFS1 were consistently associated with type 2 diabetes. In this study by Sandhu et al. there were four SNPs associated with the disease, but all were in significant linkage disequilibrium (LD) in a $39 \mathrm{~kb}$ LD block that included the H611R variant. In this study there were six UK populations and one Ashkenazi population studied - a total of approximately 
9,500 cases and 11,000 controls - yielding an odds ratio (OR) of 0.92 (95\% CI $\left.0.88-0.95, p<10^{-4}-10^{-7}\right)$, indicating once more a protective effect of the minor allele.

In this issue of the Diabetologia, two new studies attempt to replicate the previous observations. Franks et al. [7] genotyped the same four SNPs as Sandhu et al. [6] in northern Swedish Europid cases and controls. The minor allele at one SNP (rs752854) was statistically associated with reduced risk of type 2 diabetes (OR $0.85 .95 \%$ CI $0.75-0.96, p=0.010$ ), while borderline statistical significance was observed for the other three SNPs. The direction and magnitude of the associations were consistent with the previous report. Franks et. al. [7] also conducted a metaanalysis of $\sim 14,000$ cases and an equal number of controls, which included their data, together with the results of the study by Sandhu et al. [6] and unpublished data for SNPs at the WFS1 locus genotyped in three genome-wide association (GWA) studies (one Finnish, one French and one Swedish) [8-10]. This indicated that SNP rs10010131 at the WFS1 locus is associated with a reduced risk of type 2 diabetes (OR 0.89, 95\% CI 0.86-0.92; $p=4.9 \times 10^{-11}$ ).

In the second study in this issue, three of the previously studied SNPs at the WFS1 locus were genotyped in a large number $(n=3,548)$ of racially diverse members of the Diabetes Prevention Program (DPP), which was designed to compare lifestyle intervention or metformin with placebo [11]. This study differed from previous studies in that it determined the predictive value of genotypes at this locus for development of diabetes, and correlated this with different interventions. Additionally, phenotypes were obtained for all participants, including the insulin sensitivity index (reciprocal of the homeostasis model assessment of insulin resistance) and the insulinogenic index ([insulin at $30 \mathrm{~min}$-insulin at $0 \mathrm{~min}] /[$ glucose at $30 \mathrm{~min}$-glucose at $0 \mathrm{~min}]$, with insulin measured in $\mathrm{pmol} / \mathrm{l}$ and glucose in $\mathrm{mmol} / \mathrm{l})$.

The major conclusion of this well-performed study is that, overall, the WFS1 variants do not predict the onset of diabetes for the group as a whole. However, the differences in phenotypes observed in the two intervention groups may give clues as to the aetiology of the risk of diabetes conveyed by variants at this locus, i.e. impairment of beta cell function. Individuals with the variant allele H611R exhibited lower glucose-stimulated insulin secretion, yet were more insulin sensitive. Another variant was associated with reduced insulinogenic index with no difference in insulin sensitivity, suggesting that variants of WFS1 primarily affect beta cell function, consistent with patterns of expression of the gene. It is fair to say at this point as the authors do state that more detailed physiological studies need to be performed on larger samples.

\section{Why do candidate gene studies identify genes missed by GWA studies?}

There are several possible explanations. Candidate gene studies use more densely spaced SNPs to target the gene in question. More recently, because of the expense of GWA studies, candidate gene studies have included more participants and are therefore more likely to uncover lower risk alleles. Finally, in a GWA study the results are corrected for the large number of tests performed, and the threshold $p$ value for positive associations is higher, leaving room for more type 2 error. In fact, in the study by Franks et al. [7], the meta-analysis used data from GWA studies, which increased the probability that WFS1 is a risk factor for type 2 diabetes.

\section{How does WFS1 deficiency contribute to beta cell dysfunction?}

Immunocytochemistry and subcellular fractionation studies have indicated that wolframin is localised in the endoplasmic reticulum (ER) membrane and has nine transmembrane segments. Antibody studies indicate that the amino terminus extends into the cytoplasm, while the carboxy terminus is in the ER lumen [12]. Expression studies in vitro suggest that WFS1 encodes a protein with an important role in the regulation of intracellular $\mathrm{Ca}^{2+}$ homeostasis [13]. N-linked glycosylation is necessary for biogenesis and protein stability. Fibroblasts from a patient with a non-sense mutation in the gene revealed that protein absence was caused by rapid decay of the transcript, and a patient with compound missense mutations exhibited markedly reduced protein stability [12]. Thus, in patients with Wolfram syndrome mutations the protein is present at reduced levels rather than dysfunctional, although the mechanisms are completely unknown as to how the more common variants predispose/protect against type 2 diabetes.

Several studies have demonstrated a relationship between ER stress and WFS1 [14-16]. Both the mRNA and protein are induced by ER stress. Glucose-induced insulin secretion also activates WFS1 expression and wolframin production, and a reduction in WFS1 mRNA results in the activation of ER stress. ER stress as a cause of diabetes has been demonstrated in the mouse by knockout of eIF2- $\alpha$ kinase (PERK), resulting in beta cell loss and diabetes [17], and in a rare genetic disease characterised by mutation of this gene, resulting in early-onset diabetes and epiphyseal dysplasia, the Wolcott-Rallison syndrome [18]. A mouse model of Wolfram syndrome with a conditional deletion of WFS1 in beta cells revealed, by electron microscopy, a reduction in beta cell mass, enhanced apoptosis, elevation 
of a marker of ER stress and dilated ER with decreased secretory granules [14]. These results indicate that lack of expression of WFS1 in beta cells appears to be a significant contributor to the reduction in beta cell survival $[14,19]$.

\section{How can variants in WFS1 contribute to type 2 diabetes?}

The most common WFS1 variant, H611R, may be a causative variant, yet we know nothing of its potential function. Like the protective effect of the minor allele at PPARG (Pro12Ala), the minor allele at WFS1 (H611R) appears protective. In its initial phase, ER stress serves to slow down protein synthesis. During periods of starvation it might be advantageous to decrease the rate protein synthesis and reduce insulin secretion, thus accounting for the higher frequency of this variant. During periods of excess nutrition, however, this WFS1 variant may predispose to ER stress-mediated apoptosis. With regard to the five non-synonymous coding variants that were described after sequencing DNA from diabetic patients [5], any or all of which could contribute to WFS1 dysfunction, we know nothing about their potential biological effects Perhaps the best estimate of the effects of these variants can be determined by cell-based assays, yet to be performed. Additionally, these variants are in a large LD block and may be in LD with yet to be discovered regulatory variants that might alter levels of expression of this gene.

\section{What have we learned? Monogenic disease genes and multifactorial diabetes}

We now know that genes responsible for monogenic forms of diabetes are good candidates to contribute low risk for the more common forms of diabetes. These low-risk alleles require large sample sizes and rather densely spaced SNPs to be defined. When we finally identify a susceptibility locus, the biological correlates are often missing. The future lies in translating genomic observations into the contexts of gene-gene interactions, effects of environment, tissue expression, and functional biological studies.

Duality of interest The authors declare that there is no duality of interest associated with this manuscript.

\section{References}

1. Minton JA, Rainbow LA, Ricketts C, Barrett TG (2003) Wolfram syndrome. Rev Endocr Metab Disord 4:53-59
2. Polymeropoulos MH, Swift RG, Swift M (1994) Linkage of the gene for Wolfram syndrome to markers on the short arm of chromosome 4. Nat Genet 8:95-97

3. Inoue H, Tanizawa Y, Wasson J et al (1998) A gene encoding a transmembrane protein is mutated in patients with diabetes mellitus and optic atrophy (Wolfram syndrome). Nat Genet 20:143-148

4. Barrett TG (2007) Differential diagnosis of type 1 diabetes: which genetic syndromes need to be considered? Pediatr Diabetes 8 (Suppl 6):15-23

5. Minton JA, Hattersley AT, Owen K et al (2002) Association studies of genetic variation in the WFS1 gene and type 2 diabetes in U.K. populations. Diabetes 51:1287-1290

6. Sandhu MS, Weedon MN, Fawcett KA et al (2007) Common variants in WFS1 confer risk of type 2 diabetes. Nat Genet 39:951-953

7. Franks PW, Rolandsson O, Debenham SL et al (2008) Replication of the association between variants in WFS1 and risk of type 2 diabetes in European populations. Diabetologia (in press) DOI 10.1007/s00125-007-0887-6

8. Saxena R, Voight BF, Lyssenko V et al (2007) Genome-wide association analysis identifies loci for type 2 diabetes and triglyceride levels. Science 316:1331-1336

9. Scott LJ, Mohlke KL, Bonnycastle LL et al (2007) A genomewide association study of type 2 diabetes in Finns detects multiple susceptibility variants. Science 316:1341-1345

10. Sladek R, Rocheleau G, Rung J et al (2007) A genome-wide association study identifies novel risk loci for type 2 diabetes. Nature 445:881-885

11. Florez JC, Jablonski KA, McAteer J et al (2008) Testing of diabetes-associated WFS1 polymorphisms in the Diabetes Prevention Program. Diabetologia (in press) DOI 10.1007/s00125-0070891-x

12. Hofmann S, Philbrook C, Gerbitz KD, Bauer MF (2003) Wolfram syndrome: structural and functional analyses of mutant and wildtype wolframin, the WFS1 gene product. Hum Mol Genet 12:2003-2012

13. Osman AA, Saito M, Makepeace C et al (2003) Wolframin expression induces novel ion channel activity in endoplasmic reticulum membranes and increases intracellular calcium. J Biol Chem 278:52755-52762

14. Riggs AC, Bernal-Mizrachi E, Ohsugi M et al (2005) Mice conditionally lacking the Wolfram gene in pancreatic islet beta cells exhibit diabetes as a result of enhanced endoplasmic reticulum stress and apoptosis. Diabetologia 48:2313-2321

15. Ueda K, Kawano J, Takeda K et al (2005) Endoplasmic reticulum stress induces $W f S 1$ gene expression in pancreatic beta-cells via transcriptional activation. Eur J Endocrinol 153:167-176

16. Lipson KL, Fonseca SG, Ishigaki S et al (2006) Regulation of insulin biosynthesis in pancreatic beta cells by an endoplasmic reticulum-resident protein kinase IRE1. Cell Metab 4:245-254

17. Harding HP, Zeng H, Zhang Y et al (2001) Diabetes mellitus and exocrine pancreatic dysfunction in Perk-/- mice reveals a role for translational control in secretory cell survival. Mol Cell 7: $1153-1163$

18. Senee V, Vattem KM, Delepine M et al (2004) Wolcott-Rallison syndrome: clinical, genetic, and functional study of EIF2AK3 mutations and suggestion of genetic heterogeneity. Diabetes 53:1876-1883

19. Ishihara H, Takeda S, Tamura A et al (2004) Disruption of the WFS1 gene in mice causes progressive beta-cell loss and impaired stimulus-secretion coupling in insulin secretion. Hum Mol Genet 13:1159-1170 